\title{
Oil Removing from Emulsions Using Commercial Resins
}

\author{
Nattácia Rodrigues de Araujo Felipe Rocha ${ }^{1}$, Renyston Lima Ribeiro ${ }^{1}$, Nadine Pereira Merlo ${ }^{1}$ and Moilton
} Ribeiro Franco Junior ${ }^{2}$

1. Rio Verde University, Faculty of Biology and Chemistry, Rio Verde, Goiás, Brazil.

2. Federal University of Uberlândia, Chemistry Institute, Post-graduated Program in Biofuels, Uberlandia, Brazil.

\begin{abstract}
Removal of vegetal oil from wastewater using comercial resins in batch reactors processes is studied in present study. A traditional and patented type of three different surfactants was used for stabilizing of the emulsion phase. The results for the emulsion made by the synthesized surfactant showed much better stability and performance in the separation process compared to that by conventionally used Span 80. To determine the optimum operation conditions, the effect of several parameters such as initial oil concentration, amount of resin in the solid phase, adding or not a surfactant, resin type, and stabilizer concentration have been investigated. It was found that under the optimum conditions, more than $98 \%$ of oil can be removed in a single batch process.
\end{abstract}

Key words: Resins, wastewater, oil, batch.

\section{Introduction}

Oil is composed by several toxic pollutants, such as esters of fatty acids, frequently found in surface and tap waters, and in aqueous effluents from various manufacturing processes [1]. Therefore, they are hilighted in many countries in the priority list of dangerous substances discarded into the aquatic environment [2]. Since 1959 that adsorbents were first invented by Milton, 1959 [3, 4], removal of oil from wastewater has been intensively investigated using resins and activated carbon [5]. This is because the removal of oil by this method has some advantages over other separation methods [6-8].

Recently, a certain number of studies have focused on coalescence, which is an effective physical-chemical process used to destabilize and remove finely dispersed oil particles from wastewaters. Researches have shown that coalescer is effective in reducing high levels of oil and grease in appropriate particle size range that achieve technically and economically efficient oil-water separation [7-9]. Oil

Corresponding author: Nattácia Rodrigues de Araujo Felipe Rocha, M. Sc. (ormaster), reasearch field: biofuel, thermodynamics, chemistry. droplets are captured by the solid medium, forming oil films on the surfaces during the coalescence process; then the stable oil emulsions are demulsified upon continued growth of the collected oil droplets [10, 11].

The effectiveness of the coalescence process can be enhanced by using novel solid medium with special surface characteristics. Recently, researchers showed an intersting removal ability of surfactant modified adsorbents for organics or heavy metals [12, 13]. Surface modification by ion exchange with alkyl ammonium has been applied to change the packing medium surface characteristics such as polarity and hydrophobic characteristic to develop new coalescence material for oil-water separation. Ibrahim [14] reported the applicability of hexadecylpyridinium chloride treated barley straw for removal of emulsified oil in wastewater. It was found that the surfactant produced a non-polar layer on barley straw surface,which subsequently endued straw with better oil removal ability.

Many researchers [15-18] have indicated that resin modified by surfactant had satisfactory oil removal ability due to hydrogen bond formation between hydrocarbon molecules and the free hydrophobic part of the fixed surfactant. In the coalescence process, 
hydrogen bonding and hydrophobic interaction are the main driving forces for the adsorption of oil onto polymeric resins in aqueous solution [16].

Bindes and Franco have recently reported our research on treatment of wastewater containig surfactant by using activated carbon with different sizes of particle [17]. The commercial activated carbon showed an effective removing capability. Some reseachers have affirmed that it is due to the synergetic effect of cationic and hydrophobic functionalities [18, 19]. On the basis of previous research, there is a need to study the effect of surfactant modification on the surface characteristics of resin and its oil removal capability, which may further the exploration of its demulsification mechanism.

The main purpose of this paper is to illustrate the performance of the three types of resins: anionic, cationic and mixture on emulsified oily wastewater removal. Besides that, the emulsified oily wastewater was treated by commercial resin to investigate the effect of presence of one surfactant on the commercial resins oil removal performance in particular for mechanism study.

\section{Materials and Methods}

\subsection{Materials}

Polystyrene resin (cationic, anionic and mixed), obtained from Purolite-SP Sci. \& Tech. Co., is an exchange resin carrying groups with specifications close to Amberlit IRA-63. The resin was thoroughly washed in hot water and pretreated by dilute acid and base $\left(0.1 \mathrm{~mol} \cdot \mathrm{L}^{-1}\right)$ to remove impurities, then dried by vacuum pump at $25{ }^{\circ} \mathrm{C}$. Three commercial surfactants (propil betaine, Lauril sulfoccionate sodium and Lauril glucosideo) were analytical grade and purchased from EMFAL Ind. Quim. LTDA (Betim-MG) for emulsion preparation.

Vegetal soya oil and tap water were used as wetting liquids in this study, representing the oil phase and water phase, respectively. The de-ionized water was produced by self-made two stages of mixed bed ion exchange resin. Other chemicals (VETEC) were analytical grade and used without further purification.

\subsection{Oil-in-Water Emulsion}

A synthetic oil emulsion with oil concentration between 500-570 $\mathrm{mg} \cdot \mathrm{L}^{-1}$ and mean droplets diameter of $10 \mu \mathrm{m}$ was freshly prepared by mixing appropriate amount of surfactant, vegetal oil and tap water at 5,000 rpm in a blender for a time between $30 \mathrm{~min}$ and $1 \mathrm{~h}$. The oil emulsion was diluted into desired concentration as synthetic emulsified oily wastewater.

Analytical balance $\left( \pm 10^{-4} \mathrm{~g}\right)$ was used to weight the pretreated base resin (Purolite) and a desired quantity of emulsion was put into a flask reactor which was laid down under a stirrer. The three types of resins were used by adding an amount that varies from 0.1 to $0.9 \mathrm{~g}$ per reactor. The mixture was stirred in a water thermostatic bath at $25{ }^{\circ} \mathrm{C}$ for $30-60 \mathrm{~min}$; the resulting liquid phase and solid products were separated. Then, the liquid phase was analyzed in the spectrophotometer using wave length of $224 \mathrm{~nm}$. Resins were dried at $75^{\circ} \mathrm{C}$ for $5 \mathrm{~h}$ and analyzed in the VEM (Varredure Electronic Microscopy).

\subsection{Oil Removal Experimental Set-up}

The emulsified oily wastewater in feed tank was prepared into another tank and transferred to the reactor. A stirrer was working during the experiment to make the influent quality uniform. The emulsified oily wastewater was treated in resin glass batch reactor (150 mm in height and $120 \mathrm{~mm}$ in diameter) which was used as coalescer. At the bottom of the glass reactor, a rubber covered magnet plug was inserted for the stirring. The schematic diagram of the experimental set-up is shown in Fig. 1. The treatment of emulsified wastewater by different amounts of resin (cationic, anionic and mix) was carried out under same operation conditions (emulsion volume of $100 \mathrm{~mL}$, temperature of $25 \pm 1{ }^{\circ} \mathrm{C}, \mathrm{pH}=7.0$, initial oil concentration between 50 and $256 \mathrm{mg} \cdot \mathrm{L}^{-1}$ and resin mass of 0.1-0.5 
g)
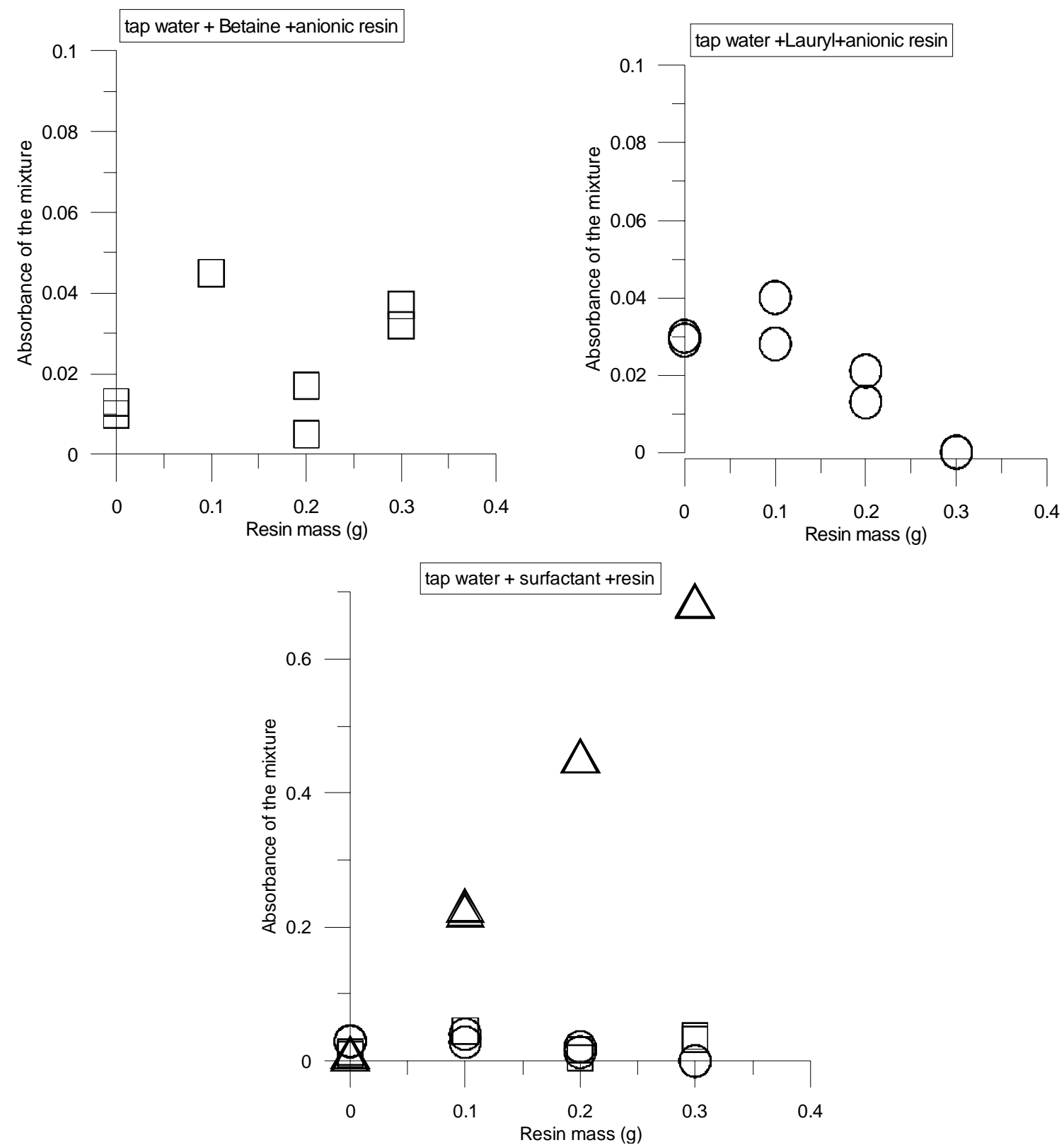

Fig. 1 Experiments using emulsion composed with surfactants + tap water without oil—influence of type of surfactant (lauryl; propil betaine) and resin (anionic: $O \square$; cationic: $\triangle$ ) in the response (absorbance).

to study the effect on oil removal capability of resins. Effluent water samples were collected after a desired coalescence interval and residual oil concentrations in solutions were determined using UV spectrophotometer (UV-2100, Shimadzu) by measuring absorbance at $224 \mathrm{~nm}$ wavelength.

\section{Results and Discussion}

Firstly, the calibration curve was built using the minimum square regression. For the absorbance in range of [0.03-1.0] and for [0.03-0.30] which shown similar results, even when samples had diluted amount of oil. Then, the fitted equation could be written as:

$$
C_{o i l}^{e q}=a \alpha_{i}^{e q}
$$

where, $C_{o i l}^{e q}$ and $\alpha_{i}^{e q}$ are the oil concentration and absorbance of the sample at equilibrium and $\alpha$ is the 
constant related to the group of points fitted. It was observed that for both experiments a was found to be $814.25 \pm 25.0$. Therefore, a plot of $C_{o i l}$ versus $\alpha_{i}$ gives a straight line of slope a and intercepts zero.

Determination of oil removed (R)

Oil was extracted from the untreated samples as initial oil concentration and final oil concentration according to the Eq. (1). Average values of analytical results were obtained from three replications. The removal percent of vegetal oil $(\% R)$ is then calculated by using the following equation:

$$
\% R=\frac{C_{i}^{o}-C_{f}^{o}}{C_{i}^{o}} 100 \%
$$

where, $C_{i}^{o}$ and $C_{f}^{o}$ are oil concentration in the emulsion at the begining and at the end of treatment, respectively.

Secondly, some experiments were carried out to analyse the behavior of the resins in the presence of surfactants without any oil. The results are depicted in Fig. 1. For anionic resin, in emulsions of propil betaine or lauryl glucoside, the biggest absorbance value observed was 0.04 even if the resin mass incresead. Otherwise, when the cationic resin was used in the experiments, it is observed that a residual or considerable amount of absorbance is measured. Also, it rises if the increasing resin mass. Therefore, cationic resin is not appropriate for removing oil from emulsions and all the experiments will be carried out using mixed and anionic resins.

For preliminary studies, the extracted oil from the studied oil-in-water emulsion samples were determined using and not using surfactants and treated with anionic resin and the results are given in Table 1. The initial oil concentration varies from around 50 to
$80 \mathrm{mg} \cdot \mathrm{L}^{-1}$ for the various emulsions and the final absorbance oil concentration varies from 0.43 to 0.60 with the percentages oil removal range from $43.0 \%$ to $60.0 \%$ Such results show the capacity and the ability of the adsorbents to strip-off the contaminant. Also, it is clear that increasing the amount of resin has not improved the percentage of oil removing.

On treating oil-in-water emulsion with initial oil concentration $56.17 \mathrm{mg} \cdot \mathrm{L}^{-1}$ using $0.2 \mathrm{~g}$ or $0.4 \mathrm{~g}$ of anionic resin has sligthly decresed the oil removal from $59.4 \%$ to $56.4 \%$. Then, increasing the initial oil concentration to $78.96 \mathrm{mg} \cdot \mathrm{L}^{-1}$ the removing slightly decreased. This reveals the importance of determining the equilibrium time, that is, when no further net adsorption occurs and the system reaches equilibrium.

Different behavior of oil removing can be observed when it was carried out experiments with the mixed resin Purolite, as can be illustrated in Table 2. It also reveals the importance of determining the optmized concentration of resin. In case of using mixed resin as the absorbent, increasing the weight of absorbent from 0.3 to $0.9 \mathrm{~g}$ led to the decrease in oil removal from $93.8 \%$ to $50 \%$ on treating sample of $65.0 \mathrm{mg} \cdot \mathrm{L}^{-1}$ (initial oil concentration) and after stirring time of 1.0 h. On treating oil-in-water emulsion sample (99.0 $\mathrm{mg} \cdot \mathrm{L}^{-1}$ initial oil concentration) with $0.4 \mathrm{~g}$ of the same resin and stirring for $1.0 \mathrm{~h}$ gave oil removal of $26.9 \%$ and with $0.6 \mathrm{~g}$ of the adsorbent and the same stirring time gives very similar result of $28.9 \%$. In the other side, diferente behavior was found when $256 \mathrm{mg} \cdot \mathrm{L}^{-1}$ of oi lis used for initial concentration, increasing the weight of absorbent from 0.4 to $0.8 \mathrm{~g}$ led to the increase in oil removal from $32.0 \%$ to $100 \%$ on treating sample after stirring time of $1.0 \mathrm{~h}$.

Table 1 Oil removal capability of anionic resin for two distinct amounts and initial oil concentration.

\begin{tabular}{llclc}
\hline & \multicolumn{5}{c}{$56.17 \mathrm{mg} \cdot \mathrm{L}^{-1}$} & $76.96 \mathrm{mg} \cdot \mathrm{L}^{-1}$ \\
\hline $\mathrm{m}_{\mathrm{R}} / \mathrm{g}$ & 0.2 & 0.4 & 0.2 & 0.4 \\
$\alpha_{f}$ & 0.028 & 0.030 & 0.055 & 0.052 \\
$\mathrm{R} \%$ & 0.594 & 0.565 & 0.433 & 0.464 \\
\hline
\end{tabular}


Table 2 Oil removal capability of mix resin for three distinct amounts and initial oil concentration.

\begin{tabular}{lllllllll}
\hline & \multicolumn{3}{c}{$65.12 \mathrm{mg} \cdot \mathrm{L}^{-1}$} & \multicolumn{3}{c}{$99.31 \mathrm{mg} \cdot \mathrm{L}^{-1}$} & \multicolumn{3}{c}{$256.41 \mathrm{mg} \cdot \mathrm{L}^{-1}$} \\
\hline $\mathrm{m}_{\mathrm{R}} / \mathrm{g}$ & 0.3 & 0.5 & 0.9 & 0.4 & 0.6 & 0.4 & 0.6 & 0.8 \\
$\alpha_{f}$ & 0.005 & 0.020 & 0.036 & 0.093 & 0.087 & 0.214 & 0.042 & 0.001 \\
$\mathrm{R} \%$ & 0.938 & 0.750 & 0.550 & 0.236 & 0.289 & 0.320 & 0.866 & 1.00 \\
\hline
\end{tabular}

\section{Conclusions}

The present study demonstrates physico-chemical properties of commercial resins produced by a brazilian industry and its importance with respect to removing of oil from emulsions containing or not one kind of surfactant. Further, its efficiency, compatibility and stability using diferents initial oil concetrations along with their additive interaction with detergents improved the washing performance. This led to conclude its future industrial application as an auxiliar of wastewater cleaning.

\section{Acknowledgements}

The authors are thankful to UniRV (Universidade de Rio Verde Project 33-2016) for financial support and Mrs. Roberta Andrade for technical support.

The Tópicos 2-PGB 634 discipline of the Chemistry Institute is duly acknowledged for helping in the discussions.

\section{References}

[1] Jeganathan, J., Nakhla, G., and Bassi, A. 2007. "Hydrolytic Pretreatment of Oily Wastewater by Immobilized Lipase.” J. Hazard. Mater. 145 (1-2): 127-35.

[2] Hua, F. L., Tsang, Y. F., Wang, Y. J., Chan, S. Y., Chua, H., and Sin, S. N. 2007. "Performance Study of Ceramic Microfiltration Membrane for Oily Wastewater Treatment.” Chem. Eng. J. 128 (2-3): 169-75.

[3] Milton, R. M. 1959. Molecular sieve adsorbents. U.S. Pat. 2882 243. filed December 24, 1953 and issued april 14, 1959.

[4] Milton, R. M. 1959. Molecular sieve adsorbents. U.S. Pat. 2882 244. filed December 24, 1953 and issued april 14, 1959.

[5] Rajakovic, V., and Skala, D. 2006. "Separation of Oil-in-water Emulsions by Freeze/thaw Method and Microwave Radiation.” Sep. Purif. Technol. 49 (2): 192-6.
[6] Price, J. M., Johnson, W. R., Marshall, C. F., Ji, Z. G., and Rainey, G. B. 2003. "Overview of the Oil Spill Risk Analysis Model for Environmental Impact Assessment.” Spill Sci. Technol. Bull. 8 (5-6): 529-33.

[7] Canizares, P., Martinez, F., Lobato, J., and Rodrigo, M. A. 2007. "Break-up of Oil-in-water Emulsions by Electrochemical Techniques.” J. Hazard. Mater. 145 (1-2) 33-240.

[8] Bratskaya, S., Avramenko, V., Schwarz, S., and Philippova, I. 2006. "Enhanced Flocculation of Oil-in-water Emulsions by Hydrophobically Modified Chitosan Derivatives, Colloids Surf.” A: Physicochem. Eng. Aspects 275 (1-3) 168-76.

[9] Li, J. Q., and Gu, Y. A. 2005. "Coalescence of Oil-in-water Emulsions in Fibrous and Granular Beds.” Sep. Purif. Technol. 42 (1): 1-13.

[10] Secerov Sokolovic, R. M., Vulic, T. J., and Sokolovic, S. M. 2006. "Effect of Fluid Flow Orientation on the Coalescence of Oil Droplets in Steady-state Bed Coalescers.” Ind. Eng. Chem. Res. 45 (11): 3891-5.

[11] Secerov Sokolovic, R. M., Vulic, T. J., Sokolovic, S. M., and Marinkovi'c Nedǔcin, R. P. 2003. "Effect of Fibrous Bed Permeability on Steady-state Coalescence.” Ind. Eng. Chem. Res. 42 (13): 3098-102.

[12] Yang, B. W., and Chang, Q. 2008. "Wettability Studies of Filter Media using Capillary Rise Test.” Sep. Purif. Technol. 60 (3): 335-40.

[13] Jegatheesan, V., and Vigneswaran, S. 1997. “The Effect of Concentration on the Early Stages of Deep Bed Filtration of Submicron Particles.” Water Res. 31 (11): 2910-13.

[14] Dabrowski, A., Podko’scielny, P., Hubicki, Z., and Barczak, M. 2005. "Adsorption of Phenolic Compounds by Activated Carbon-A Critical Review.” Chemosphere 58 (8): 1049-70.

[15] Pangu, G. D., and Feke, D. L. 2004. "Acoustically Aided Separation of Oil Droplets from Aqueous Emulsions.” Chem. Eng. Sci. 59 (15): 3183-93.

[16] Ibrahim, S., Wang, S., and Ang, H. M. 2010. "Removal of Emulsified Oil from Oily Wastewater Using Agricultural Waste Barley Straw.” Biochem. Eng. J. 49 (1): 78-83.

[17] Bindes, M. M. M., and Franco Jr, M. R. 2015. “Surfactant 
Removal from Aqueous Solutions onto Activated Carbon Using UV Spectroscopy.” Des. and Water Treat 56 (11): 2890.

[18] Roques, H., and Aurelle, Y. 1991. "Oil-water Separations Oil Recovery and Oily Wastewater Treatment.” In New Developments in Industrial Wastewater Treatment, edited by Uslu, O. Netherlands: Kluwer Academic Publisher, 155-74.

[19] Lei, Z., and Zhang, L. 2008. "Morphological Evolution of Aggregates Induced by the Hydrophobic Effect and Hydrogen Bonding Interaction.” Colloid Surf. A 312 (2-3): 166-71. 